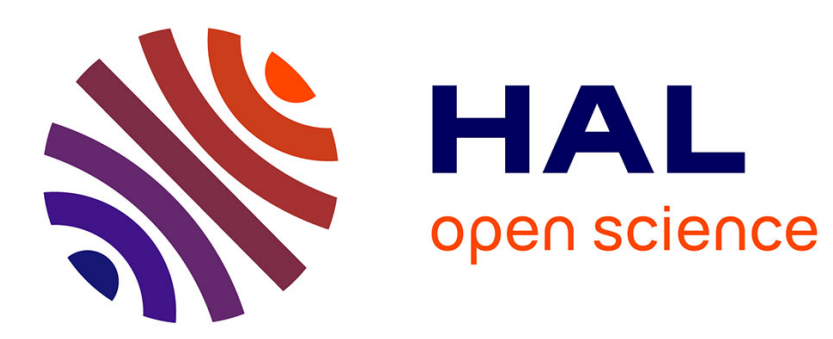

\title{
Effet d'écran dans une salle et méthode de perturbation
}

\author{
D. Habault
}

\section{To cite this version:}

D. Habault. Effet d'écran dans une salle et méthode de perturbation. Journal de Physique IV Proceedings, 1994, 04 (C5), pp.C5-109-C5-112. 10.1051/jp4:1994517 . jpa-00252850

\section{HAL Id: jpa-00252850 https://hal.science/jpa-00252850}

Submitted on 1 Jan 1994

HAL is a multi-disciplinary open access archive for the deposit and dissemination of scientific research documents, whether they are published or not. The documents may come from teaching and research institutions in France or abroad, or from public or private research centers.
L'archive ouverte pluridisciplinaire HAL, est destinée au dépôt et à la diffusion de documents scientifiques de niveau recherche, publiés ou non, émanant des établissements d'enseignement et de recherche français ou étrangers, des laboratoires publics ou privés. 


\title{
Effet d'écran dans une salle et méthode de perturbation
}

\author{
D.M.L. HABAULT
}

C.N.R.S., Laboratoire de Mécanique et d'Acoustique, 31 chemin Joseph Aiguier, 13402 Marseille cedex 20, France

\begin{abstract}
The aim of this paper is to investigate the interest of a perturbation method to describe a screen effect in a room. The screen is described as an elastic plate and can vibrate. First, the sound pressure in the room is computed by using a boundary element method. Then, a perturbation method is developed taking advantage of a small parameter related to the ratio of the densities of the fluid and the plate. Numerical simulations are presented to examine the convergence of the method.
\end{abstract}

\section{INTRODUCTION.}

On étudie ici l'intérêt d'une méthode de perturbation pour calculer la pression émise dans une salle en présence d'un écran. L'écran est modélisé par une plaque mince élastique. La pression est calculée, au paragraphe 2, par une méthode "exacte". d'équations intégrales et, au paragraphe 3, par une méthode de perturbation [1,2] qui permet de réduire notablement les temps de calcul. L'exemple traité est bidimensionnel et la salle est rectangulaire pour simplifier le traitement numérique. Les simulations sont présentées pour trois types de matériaux afin de donner un exemple des avantages et limites de la méthode.

\section{METHODE D'EQUATIONS INTEGRALES.}

Dans la salie rectangulaire, une source placée en un point $S$ émet un signal périodique en temps (exp(-iut), $\omega$ étant la pulsation). L'écran est modélisé par une plaque élastique mince. La pression émise dans la salle est calculée en résolvant le problème de couplage entre la plaque et le fluide. Le fluide a pour masse volumique $\rho_{f}$. La plaque, notée $\Sigma$, est caractérisée par un module d'Young $E$, un coefficient de Pọisson $v$, une épaisseur $h$ et une masse volumique $\rho_{p}$. Les quatre parois de la salle, $B_{j}$, sont caractérisées par des coefficients de réflexion $\beta_{j}$. Les points $L^{-}$et $L^{+}$ sont les extrémités de la plaque. Un point $M^{\prime}$ de la plaque est repéré par son abscisse s' sur la plaque. p, la pression dans la salle, et $u$, le déplacement sur la plaque, sont solutions du système suivant : 


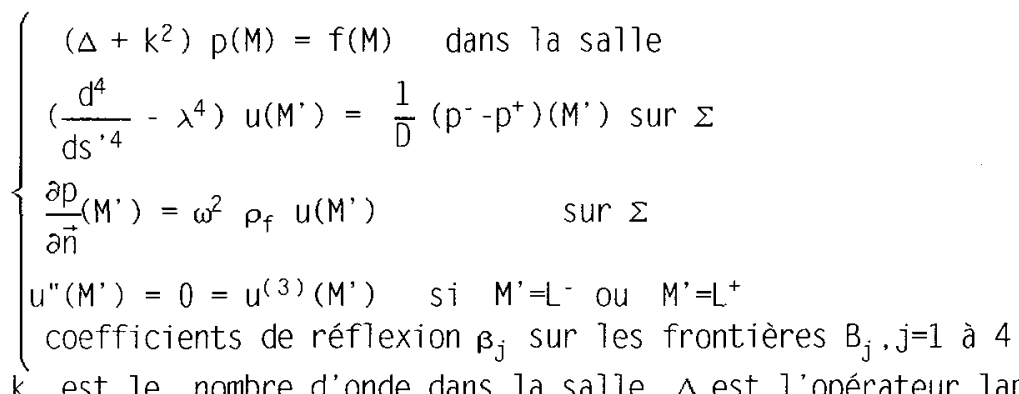
source acoustique. $D$ est la rigidité de la plaque : $D=E h^{3} / 12\left(1-v^{2}\right)$ et $\lambda^{4}$ est donné par $\lambda^{4}=\rho_{p} h \omega^{2} / 0$. Le terme $\left(p^{-}-p^{+}\right)$représente la discontinuité de $p$ à la traversée de $\Sigma$. Les conditions (1-c) correspondent à la continuité des vitesses normales sur $\Sigma$ et les conditions (1-d) à des conditions "bords libres".

$p$ peut alors s'écrire sous la forme intégrale [3]:

$$
P(M)=p_{\text {in }}(M)+\int_{\Sigma} \mu(P) \frac{\partial G(M, P)}{\partial \vec{n}(P)} d \sigma(P)
$$

où $G$ est le noyau de Green pour la salle vérifiant les mêmes conditions aux limites que $p$ sur les frontières $B_{j}$. $p_{i n}=G^{*} f$ est donc la pression incidente (en $l^{\prime}$ 'absence d'écran). $\mu, l^{\prime}$ inconnue, est égale au saut de pression $\left(p^{+}-p^{-}\right)$à la traversée de $\Sigma$. on montre que $u$ admet également une représentation intégrale :

$$
u\left(M^{\prime}\right)=-\int_{\Sigma} \mu\left(M^{\prime},\right) \Gamma\left(M^{\prime}, M^{\prime}{ }^{\prime}\right) d s^{\prime}+\vec{s} \cdot \overrightarrow{\mathcal{G}}\left(M^{\prime}\right)
$$

$\Gamma$. le noyau de Green pour la plaque infinie, est connu explicitement. Le vecteur $\vec{s}$, à déterminer, représente les discontinuités de $u$ et de sa dérivée première en $\mathrm{L}^{-}$et $\mathrm{L}^{+}$, et 1 e vecteur $\overrightarrow{\mathcal{G}}$, connu, s'exprime en fonction des dérivées d'ordre 2 et 3 de $\Gamma$ aux deux extrémités de la plaque.

L'écriture de la continuité des vitesses normales sur $\Sigma$ conduit à 1 'équation intégrale:

$$
\begin{aligned}
& \frac{\partial P_{i n}}{\partial \vec{n}}(P)-P_{P^{\prime \prime} \rightarrow P} \frac{\partial}{\partial \vec{n}} \int_{\Sigma} \mu\left(P^{\prime}\right) \frac{\partial G\left(P^{\prime \prime}, P^{\prime}\right)}{\partial \vec{n}\left(P^{\prime}\right)} d \sigma\left(P^{\prime}\right)= \\
& =\omega^{2} \rho_{f}\left\{-\int_{\Sigma} \mu\left(M^{\prime}\right) \Gamma\left(P, M^{\prime}\right) d s^{\prime}+\vec{s} \cdot \overrightarrow{\mathcal{G}}(P)\right\}
\end{aligned}
$$

où les inconnues sont la fonction $\boldsymbol{\mu}$ et le vecteur $\vec{s}$. Elles sont obtenues en ajoutant à l'équation (4) les quatre équations provenant des conditions (1-d) où l'on introduit u sous sa forme intégrale (3). Le système obtenu est résolu par une méthode de collocation classique. La pression $p$ en tout point de la salle est ensuite calculée à l'aide de (2).

\section{METHODE DE PERTURBATION.}

Dans 1'équation (4), apparaît naturellement le paramètre $\varepsilon=\rho_{f} / \rho_{p} h$. Si ce paramètre est petit, T'équation (4) peut alors être résolue par une méthode de perturbation, c'est-à-dire en remplaçant les inconnues $\boldsymbol{\mu}$ et $\overrightarrow{\mathcal{S}}$ par les premiers termes d'un développement en $\varepsilon^{j}$

$$
\mu=\mu^{0}+\varepsilon \mu^{1}+O\left(\varepsilon^{2}\right) \quad ; \quad \vec{S}=\vec{S}^{0}+\varepsilon \vec{S}^{1}+O\left(\varepsilon^{2}\right)
$$

En introduisant la notation $v$ telle que : $v=\omega^{2} \rho_{p}$ hu et le développement de $v$ en fonction de $\varepsilon$, on montre que les premiers termes de ces développements sont 
solutions des équations :

$$
\begin{aligned}
& \int_{\Sigma} \mu^{0}(P) K(M, P) d \sigma(P)=\frac{\partial p_{i n}}{\partial \vec{n}}(M) \quad \text { sur } \Sigma \\
& V^{0}(M)=-\int_{\Sigma} \mu^{0}(P) r(M, P) d \sigma(P)+\vec{S}^{0} \cdot \vec{G}_{\gamma}(M) \\
& \int_{\Sigma} \mu^{1}(P) K(M, P) d \sigma(P)=v^{0}(M) \quad \text { sur } \Sigma
\end{aligned}
$$

où : $\quad \quad \quad \quad(M, P)=\rho_{p} h \omega^{2} \Gamma(M, P), \vec{g}_{\gamma}$ s'exprime en fonction de $r$ et

$$
K(M, P)=\frac{\partial}{\partial \vec{n}(M)} \frac{\partial}{\partial \vec{n}(P)} G(M, P) \text { le noyau de } 1 \text { 'équation intégrale. }
$$

L'équation (5) est une équation intégrale pour $T^{\prime}$ 'inconnue $\boldsymbol{\mu}^{0}$. Elle correspond au problème de diffraction par un écran rigide. Elle est résolue par collocation comme précédemment. La relation (6) permet d'obtenir quatre équations pour déterminer $\vec{\delta}^{0}$ puis de calculer $v^{0}$ en tout point de la plaque. L'équation (7) est alors une équation intégrale pour $\mu^{1}$, possédant le même noyau que 1 'équation (5) ; la matrice correspondant au système linéaire obtenu par la méthode de collocation est donc la même que pour l'équation (5), seut change le second membre, ce qui conduit à des temps de calcul très réduits. L'approximation de la pression peut alors être obtenue par la représentation intégrale (2) où $\mu$ est remplacé par les deux premiers termes de son développement :

$$
p(M) \simeq\left(p^{0}+\varepsilon p^{1}\right)(M)=p_{i n}(M)-\int_{\Sigma}\left(\mu^{0}+\varepsilon \mu^{1}\right)(P) \frac{\partial G(M, P)}{\partial \vec{n}(P)} d \sigma(P)
$$

L'approximation de $p$ à l'ordre 0 correspond à la pression émise dans la salle lorsque T'écran est parfaitement réfléchissant.

Les figures 1 à 3 présentent une comparaison de niveaux sonores dans la salle obtenus par la méthode d'équations intégrales (1igne continue) puis la méthode de perturbation à 7 'ordre 0 (pointillés) et 1 (croix). Les courbes ont été calculées à $100 \mathrm{~Hz}$, pour une salle de dimensions $25 * 9 \mathrm{~m}^{2}$, pour un écran horizontal suspendu au plafond, en fonction de la distance horizontale source-écran. Les valeurs de $\varepsilon$ sont respectivement : 0.033 (fig. 1), 0.17 (fig. 2), 0.40 (fig. 3). Sur la fig. 1, le matériau est de l'acier : 1'approximation "écran rigide" est déjà tout à fait. suffisante ; sur la fig. 3. le matériau (contre-plaqué) est trop léger. le coefficient $\varepsilon$ est trop grand pour avoir une bonne approximation. Sur la fig. 2, pour une valeur intermédiaire de $\varepsilon$, 1'approximation à 1 'ordre 1 est notablement meilleure que celle à l'ordre 0 .

\section{REFERENCES :}

[1] Filippi P.J.T., Lagarrigue 0. and Mattéi P.0., Perturbation method for sound radiation by a vibrating plate in a light fluid: comparison with the exact solution, accepté pour publication au J.S.V.

[2] Crighton D., The 1988 Rayleigh lecture : fluid loading - the interaction between sound and vibration, J.S.V., 133(1) (1989) 1-27.

[3] Filippi P.J.T., Layer potentials and acoustic diffraction, J.S.V., 54(4) (1977) 473-500. 

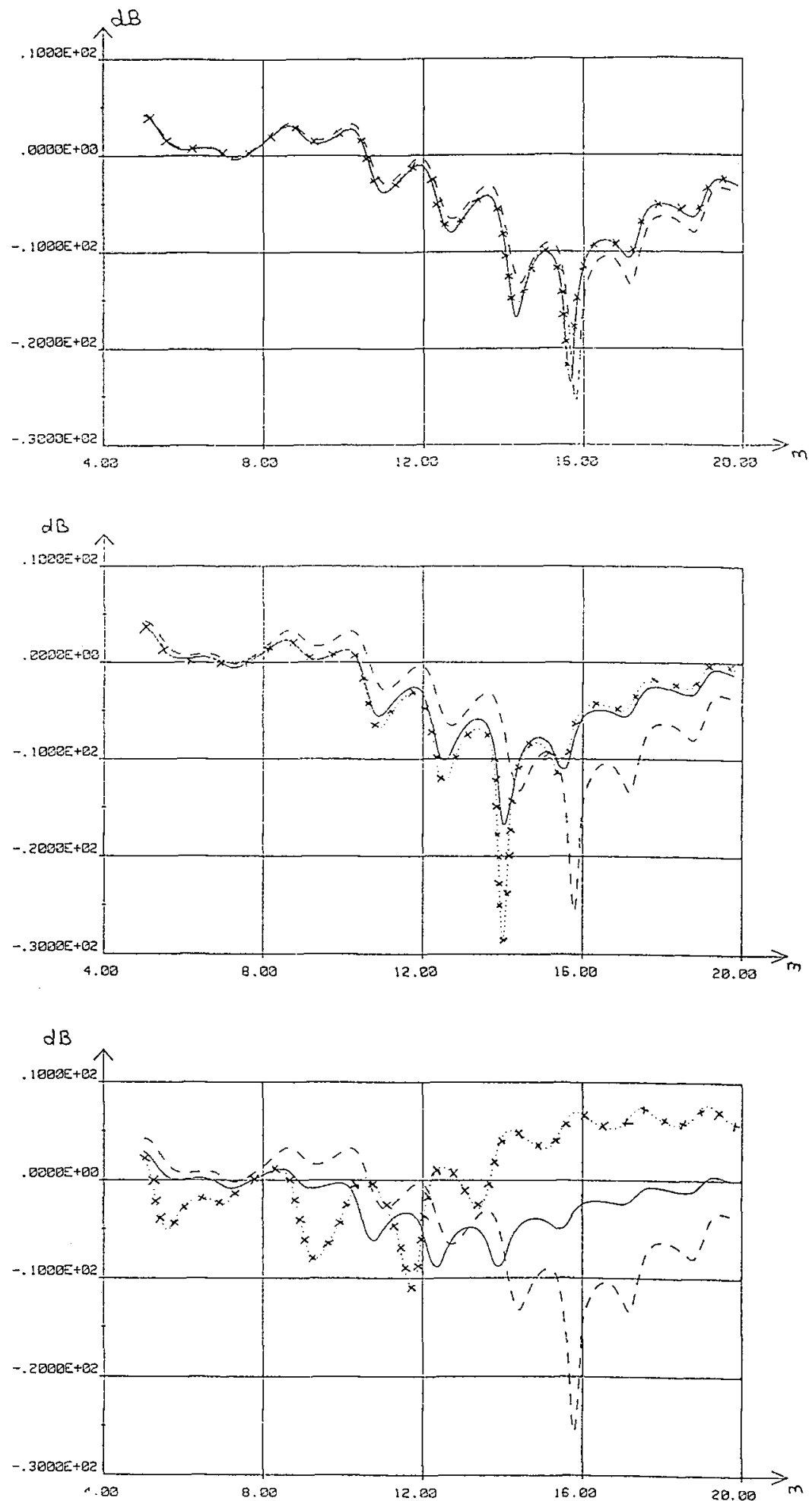